Grenzen des Politischen 
Stephan Lahrem/Olaf Weißbach

\title{
Grenzen des Politischen
}

\author{
Philosophische Grundlagen \\ für ein neues politisches Denken
}

Verlag J. B. Metzler

Stuttgart - Weimar 
Die Deutsche Bibliothek - CIP-Einheitsaufnahme

Lahrem, Stephan: Grenzen des Politischen :

philosophische Grundlagen für ein neues politisches Denken /

Stephan Lahrem ; Olaf Weißbach. -

Stuttgart ; Weimar : Metzler, 2000

ISBN 978-3-476-01753-6

Dissertationssigle der FU Berlin D 188

ISBN 978-3-476-01753-6

ISBN 978-3-476-01715-4 (eBook)

DOI $10.1007 / 978-3-476-01715-4$

Dieses Werk einschließlich aller seiner Teile ist urheberrechtlich geschützt. Jede Verwertung außerhalb der engen Grenzen des Urheberrechtsgesetzes ist ohne Zustimmung des Verlages unzulässig und strafbar. Das gilt insbesondere für Vervielfältigungen, Übersetzungen, Mikroverfilmungen und die

Einspeicherung und Verarbeitung in elektronischen Systemen.

(C) 2000 Springer-Verlag GmbH Deutschland

Ursprünglich erschienen bei J.B. Metzlersche Verlagsbuchhandlung und Carl Ernst Poeschel Verlag GmbH in Stuttgart 2000 


\section{Inhaltsverzeichnis}

Vorwort $\ldots \ldots \ldots \ldots \ldots \ldots \ldots \ldots \ldots \ldots \ldots, \ldots \ldots$, VII

Methode und Gegenstand $\ldots \ldots \ldots \ldots \ldots \ldots \ldots \ldots \ldots \ldots \ldots$

Grenzbestimmung als Methode $\ldots \ldots \ldots \ldots \ldots \ldots \ldots \ldots \ldots$

Grenzen der Dialektik ..................... 8

Die Kategorie der Besonderheit ............... 10

Grenzen der Dialektik .................... 21

Der Idealtypus als Erkenntnismittel der Besonderheit ...... 39

Der Idealtypus in seiner Bedeutung als Grenzbegriff .... 40

Zweck und Konstruktionsprinzipien des Idealtypus ..... . 46

Max Webers Idealtypus und unsere Fragestellung ...... 58

Begriffsbildung und Begriffe des Politischen ............ 62

Die Begriffsbildung und der Begriff des Politischen

bei Carl Schmitt .......................... 64

Konkrete Begriffsbildung ................. 66

Die erkenntnistheoretische Bedeutung der Ausnahme ... 73

Kategorien des Politischen .................. 77

Die Begriffsbildung des Politischen

bei Hannah Arendt ..................... 81

Die Originalität des Phänomens totaler Herrschaft . . . . 84

Natalität: die zentrale Kategorie des Politischen ........ 95

Heilmittel gegen die Aporien des Handelns . . . . . . . . 105

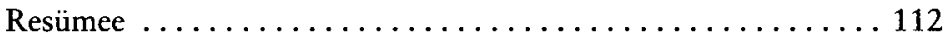

Grenzen des Politischen - Das Problem der Eigenständigkeit . . . 119

Wissenschaft und Politik ...................... 122

Das platonische Experiment .................. 125

Rationalisierung der Politik .................. 136

Ökonomie und Politik ......................... 139

Das Versprechen der Arbeit auf Freiheit ............ 142

Die soziale Frage als Inhalt der Politik ............ 145

Die Öffnung des Politischen für die Ökonomie ........ 153 
Recht und Politik ........................ 158

Gewalt und Gegengewalt.................... 160

Die Verrechtlichung der Politik . . . . . . . . . . . 170

Religion und Politik ..................... 183

Die politische Neutralisierung der Religion .......... 190

Das Verhältnis von Religiösem und Politischem ........ 199

Ästhetik und Politik ....................... 204

Die Besonderheit als zentrale Kategorie der Ästhetik . . . . 207

Die Politisierung des Ästhetischen und

die Ästhetisierung des Politischen .............. 215

Resümee .................................. 227

Grenzen des Politischen - Das Problem der Maßlosigkeit ..... 231

Individualisierung und Universalisierung

in der Massendemokratie . .................. 233

Der Begriff Massendemokratie ............... 237

Die politischen Leidenschaften in der Massendemokratie . 244

Politischer Avantgardismus ................... 258

Die geschichtsphilosophische Begründung der Avantgarde . . 262

Die Demokratisierung des Avantgardekonzeptes ....... 270

Kulturelle Ressourcen für die Hegung der Maßlosigkeit

des Politischen .......................... 280

Leidenschaft und Politik ................... 281

Die Sphäre der Privatheit .................... 288

Die Sphäre der Öffentlichkeit ................ 299

Politische Institutionen und das Problem der Bürokratisierung .. 305

Zum Begriff der Institution . . . . . . . . . . . . . 307

Das Verbältnis von Politik und Institution ........... 313

Die Stellung der Bürokratie im und zum Politischen .... 318

Zum Problem der Gewaltenteilung .............. 325

Resümee ............................... 333

Das `hölzerne Eisen` politische Philosophie ............. 339

Literaturverzeichnis $\ldots \ldots \ldots \ldots \ldots \ldots \ldots \ldots \ldots \ldots \ldots \ldots \ldots \ldots$






\section{Vorwort}

Das politische Denken sieht sich am Ende des 20. Jahrhunderts mit einer neuen Situation konfrontiert, die sich durch einen unmittelbaren Rückgriff auf die Theoriebestände des Liberalismus und des Kommunismus, deren Kampf dieses Jahrhundert geprägt hat, nicht angemessen begreifen läßt. Denn vom Zusammenbruch der kommunistischen Staatenwelt ist auch die sie tragende Ideologie des Marxismus betroffen. Mag der Traum von einer gerechten Welt auch weiterhin Bestand haben, so ist doch der Versuch, dieses Ziel im Verbund von Wissenschaft, Ökonomie und Politik herzustellen, so tiefgreifend gescheitert, daß die Aufführung des platonischen Experiments in dieser Form als enttäuscht gelten kann. Aber auch die Theorie des Siegers im Weltbürgerkrieg, der Liberalismus, ist nicht unbeschädigt geblieben. Nicht nur wurde die Hoffnung auf ein erreichtes Ende der Geschichte in Jugoslawien zerstört; gerade innerhalb der westlichen Massendemokratien wird immer deutlicher, daß die liberale Vergesellschaftung im Namen individueller Freiheit die politischen Institutionen der repräsentativen Demokratie zunehmend schwächt, die die Voraussetzung jener Freiheit sind.

Wenn heute von einem Verlust von Utopien gesprochen wird, der auch den Glauben an die politische und soziale Kraft eines technischen Fortschritts einschließt, so wird die Situation für die politische Philosophie noch prekärer, weil in unseren Augen nicht nur die Vorstellung von der Herstellbarkeit einer guten und gerechten Welt in die Krise geraten ist und der politische Avantgardismus unter die Ursachen dieser Krise gerechnet werden muß; auch der Rückgriff auf eine geschichtsphilosophisch begründete Substanz des Politischen, die den normativen Maßstab zur Beurteilung politischer Phänomene abgibt, gilt uns als durch die Geschichte selbst widerlegt.

Die Reformulierung einer politischen Philosophie, der das Vertrauen in ein übergeschichtliches Allgemeines abhanden gekommen ist und die sich zugleich von dem Glauben abwendet, ein zukünftiges Allgemeines herstellen zu können, muß daher auf dem schwankenden Boden des Relativismus als einer nicht zu ermäßigenden Voraussetzung erfolgen. Die politische Philosophie wird diese Herausforderung nicht meistern können, wenn sie nicht - so unsere These - ihre Orientierung an einem 
Allgemeinen oder einem verallgemeinerbaren Einzelnen aufgibt, sich der Kategorie Besonderheit zuwendet und die historische Selbstartikulierung der Gesellschaft als Ausgangspunkt der philosophischen Reflexion anerkennt. Eine solche Orientierung an der Besonderheit empfiehlt sich unseres Erachtens noch aus einem zweiten Grund.

Will man - und dies ist unsere, durch die Wissenschaft selbst nicht zu legitimierende Wertentscheidung - einer der grundlegenden Erfahrungen des 20. Jahrhunderts, der Erfahrung totaler Herrschaft, philosophisch Rechnung tragen, dann sieht sich die politische Philosophie vor der Herausforderung, Kategorien und eine spezifische Art der Begriffsbildung bereitzustellen, die ein nicht-totalitäres politisches Denken erlauben. Begreift man totale Herrschaft ganz allgemein als eine Entgrenzung des Politischen - ganz gleich ob man sie als eine vollständige Entpolitisierung des Politischen versteht oder im Gegenteil als eine Verabsolutierung der Politik -, so ist nach den Grenzen des Politischen zu fragen, nach der Besonderheit dieser Sphäre, in der politisch gehandelt und entschieden wird.

Die im Titel dieses Buches formulierte Frage nach den Grenzen des Politischen wie die Frage nach dem Status der Kategorie Besonderheit für die politische Philosophie sind die beiden zentralen methodischen Orientierungen bei unserem Versuch der Reformulierung einer politischen Philosophie, die die Ressourcen für eine Hegung der Maßlosigkeit des Politischen sichtbar machen kann.

Für ein solches Vorhaben war es zunächst notwendig, Methoden der Grenzbestimmung in den Blick zu nehmen. Mit der komplementären Verschränkung von zwei methodischen Ansätzen - den Grenzen der Dialektik und Max Webers idealtypischer Begriffsbildung - stellen wir eine neuartige Methode politischen Denkens vor. Sie zielt auf die Erkenntnis der Besonderheit, sowohl kategoriell wie hinsichtlich der historischen Individualität. Im zweiten Teil unseres einleitenden Abschnitts haben wir den Gegenstandsbereich, das Politische, mit Hilfe der beiden paradigmatischen Ansätze von Carl Schmitt und Hannah Arendt gesichtet. Aufgrund der im Abschnitt >Methode und Gegenstand versammelten methodischen und begrifflichen Werkzeuge sind wir im zweiten Abschnitt unserer Arbeit an die systematische Bestimmung der Grenzen des Politischen gegangen.

Dabei war zunächst die Besonderheit des Politischen, die Eigenständigkeit dieser Sphäre zu begründen, ohne auf eine irgendwie geartete Substanz des Politischen zurückzugreifen. Nach der Untersuchung der Wirkungsweise mit anderen Bereichen menschlichen Lebens ließ sich das Resultat paradox formulieren: Die Herausbildung des Politi- 
schen beruht auf der aporetischen Realisierung der in den anderen Bereichen menschlichen Lebens geltenden Wertmaßstäbe; erst durch deren Wirklichkeit, dem gegenständlichen Wirken der ihnen inhärenten Kräfte, wird das Politische als eigenständige Sphäre hervorgebracht. In dieser Angewiesenheit bleibt das Politische notwendig unbestimmt, denn um seines eigenen Geltungsanspruchs willen läßt sich ein Handeln im Politischen auf keinen ihm äußeren, vorausgesetzten Maßstab normativ verpflichten. Gerade deshalb wird der Politik die Neutralisierungsleistung der konfligierenden Potenzen zugetraut und ihr für diese ihr eigentümliche Aufgabe alle nötigen Kompetenzen und Mittel zugesprochen. Diese Zumutung, der maßgebende Bereich menschlichen Lebens unter der Voraussetzung der Freiheit von allen Maßstäben zu sein, bedeutet zugleich eine Ermächtigung der Politik, insofern sie die Freiheit zur Anwendung aller möglichen Maßstäbe einschließt.

Das sich aus der Maßstablosigkeit ergebende Problem der Freiheit haben wir daher im dritten Abschnitt als Problem der prinzipiellen Maßlosigkeit politischen Handelns diskutiert. Will man nicht darauf verzichten, die stets präsente Bedrohung der Maßlosigkeit zu bannen und die Erfahrungen dieses Jahrhunderts mit totalitärer Politik und dem Einsatz der Atombombe sind für uns Anlaß genug, darauf nicht verzichten zu wollen -, und zugleich auf der Höhe des Problems bleiben, schien es uns angemessen, einen Perspektivenwechsel vorzunehmen und nach den Möglichkeiten und Bedingungen für eine Hegung des Politischen zu fragen. Aufgrund der damit verbundenen Inversion der Fragestellung, die die Grenzen des Politischen von innen zu bestimmen sucht, haben wir kulturelle Ressourcen und die Orte ihrer Entstehung aufzuzeigen versucht, die eine Haltung von 'Leidenschaft und Augenmaß ermöglichen und somit der prinzipiellen Maßlosigkeit politischen Handelns entgegenwirken. Dabei zeigte sich ein zweites Mal - diesmal vom Standpunkt des Politischen und um seines Erhaltes willen - die Angewiesenheit und Bedingtheit des Bereichs des Politischen auf ihm nicht verfügbare Voraussetzungen. Da diese selbst historischer Art sind, war nach dem Charakter der politischen Leidenschaften unter den gegenwärtigen Bedingungen der Massendemokratie zu fragen und danach, ob diese der Hegung des Politischen förderlich sind oder nicht.

In diesem Buch haben wir versucht, die Grundlagen für eine Reformulierung politischer Philosophie zu erarbeiten, die den Erfahrungen unseres Jahrhunderts sowohl kategoriell wie hinsichtlich der Art der Begriffsbildung Rechnung trägt. Da sich eine politische Philosophie, die an der Bestimmung der Grenzen des Politischen interessiert ist, nicht mit dem Aufweis des zentralen Stellenwerts der Kategorie Be- 
sonderheit und der Bereitstellung der angemessenen Methoden erschöpfen kann, haben wir im Schlußkapitel einige Kategorien des Politischen vorgestellt, die der Besonderheit inhaltlich und strukturell Rechnung tragen. Solche Kategorien - von denen wir beispielhaft Machtkampf, Entscheidung und Repräsentation im Schlußkapitel skizziert haben - nennen wir operative Kategorien der Besonderheit. Operativ ist hier zu verstehen im Sinne des Weberschen Idealtypus, das heißt, solche Kategorien bilden das Instrumentarium zur Beurteilung der konkreten gesellschaftlichen Wirklichkeit und genauer: Indem man die konkrete Wirklichkeit mit diesen Kategorien vergleicht, kann man Aussagen darüber formulieren, inwieweit in einer Gesellschaft die Bedingungen und Ressourcen für eine Hegung des Politischen gegeben bzw. nicht gegeben sind. Diese sichtbar zu machen und damit ein Bewußtsein des Politischen zu fördern, ist die erklärte Absicht unserer Arbeit.

Damit ist zugleich angezeigt, was eine politische Philosophie, die auf die Bestimmung der Grenzen des Politischen zielt, nicht leisten kann und nicht leisten will. Von einer solchen politischen Philosophie ist, gerade weil sie die Kategorie Besonderheit ins Zentrum ihrer Reflexionen stellt, kein theoretisches System zu erwarten. Die Kategorie Besonderheit sperrt sich gegen die Vorstellung, eine Zentralkategorie als archimedischen Punkt bilden zu können, von dem aus sich ein Kategoriensystem des Politischen entfalten ließe. Noch weniger ist von dieser politischen Philosophie ein normativer Bezugspunkt für praktisches Handeln, gar ein Politikmodell zu erwarten. Gerade wenn die politische Philosophie Anspruch auf Wirklichkeitswissenschaft erhebt, d. h. wenn sie ihre Bedingtheit durch die Historizität des Politischen hervorhebt und nicht die Unbedingtheit des Denkens vertritt - gerade dann muß die politische Philosophie den Legitimationszusammenhang von Theorie und Praxis bestreiten. Politische Philosophie ist wesentlich Nach-Denken. Wer von ihr ein Vor-Denken erwartet, überschätzt nicht nur ihre Leistungsfähigkeit, sondern beschädigt sie. Denn auch für sie gilt, daß sie ihre Stärke nur erhalten und entfalten kann, wenn sie ihre eigenen Grenzen anerkennt. Damit wird deutlich, daß unsere Intention, Methoden und Kategorien für ein nicht-totalitäres politisches Denken bereitzustellen, nicht nur eine politische Dimension hat, sondern wesentlich philosophischer Art ist. Mit unserer Arbeit zielen wir auf eine Reformulierung politischer Philosophie, die ihre Stärke daraus gewinnt, daß sie die Grenzen ihres Reflexionsvermögens anerkennt.

Ob wir diesen Ansprüchen gerecht geworden sind, darüber wird nicht zuletzt der Leser entscheiden. Wenn wir ihm gelegentlich, gerade 
in unseren methodischen Überlegungen, Beharrlichkeit abverlangen, so waren wir doch darauf bedacht, daß unser Gedankengang stets nachvollziehbar und unsere Sprache bei aller kategoriellen Schärfe jederzeit verständlich bleibt. So hoffen wir, mit unserer Arbeit nicht nur bei denjenigen auf Interesse zu stoßen, die sich mit der politischen Philosophie von Berufs wegen auseinandersetzen.

Das vorliegende Buch ist die gemeinsam verfaßte Dissertation, die wir im Sommer 1998 am Institut für Philosophie der Freien Universität Berlin eingereicht haben. Für die Veröffentlichung haben wir nur geringfügige Änderungen vorgenommen. $\mathrm{Da}$ wir die Arbeit in dieser Form vorlegen können, verdanken wir zuallererst unserem Lehrer und Doktorvater Prof. Dr. Peter Furth. Er hat uns in den vergangenen Jahren in zahllosen Gesprächen mit Rat zur Seite gestanden. Vor allem hat er uns stets dazu angehalten, im Denken keine Kompromisse einzugehen, uns nicht mit bequemen Einsichten zufriedenzugeben und uns zugleich davor gewarnt, diese Kompromißlosigkeit auf den Bereich der Politik zu übertragen. Von diesem Plädoyer für eine Freiheit des Denkens und für eine Maßhaltung im Politischen ist diese Arbeit geprägt. Unser Dank gilt auch unserem zweiten Gutachter, dem Privatdozenten Dr. Bernhard Heidtmann, der in vielen Diskussionen unser Bewußtsein für die Theorie bürgerlicher Gesellschaft, für ihre Probleme und ihre Stärken, geschärft hat. Von denjenigen, die unsere Arbeit in den letzten Jahren mit Anregungen und Kritik begleitet haben, wollen wir hier stellvertretend den Freund und Kollegen Christian Schlüter nennen. Und nicht zuletzt wollen wir der Nachwuchsförderung des Landes Berlin danken, die uns ein zweijähriges Promotionsstipendium gewährt hat.

Berlin, September 1999

Stephan Lahrem/Olaf Weißbach 